\title{
Combining the Ultra-Weak Variational Formulation and the Multilevel Fast Multipole Method*
}

\author{
Eric DARRIGRAND \\ IRMAR - Université de Rennes 1 \\ Campus de Beaulieu \\ 35042 Rennes cedex, France \\ eric.darrigrand-lacarrieu@univ-rennes1.fr
}

\author{
Peter MonK \\ Department of Mathematical Sciences \\ University of Delaware \\ Newark, DE 19716, USA \\ monk@math.udel.edu
}

January 4, 2011

\begin{abstract}
Because of its practical significance, many different methods have been developed for the solution of the time-harmonic Maxwell equations in an exterior domain at higher frequency. Often methods with complimentary strengths can be combined to obtain an even better method. In this paper we provide a numerical study of a method for coupling of the Ultra-Weak Variational Formulation (UWVF) of Maxwell's equations, a volume based method using plane wave basis functions, and an overlapping integral representation of the unknown field to obtain an exact artificial boundary condition on an auxiliary surface that can be very close to the scatterer. Combining the new algorithm with a multilevel fast multipole method we obtain an efficient volume based solver with an exact auxiliary boundary condition, but without the need for singular integrals.
\end{abstract}

Keywords

Ultra-Weak Variational Formulation - ABC - Integral Representation - Multilevel Fast Multipole Method

\section{Introduction}

At progressively higher frequencies, the problem of approximating the time-harmonic Maxwell equations in an exterior domain is increasingly challenging because of the need for an increasingly large number of unknowns per wavelength to maintain accuracy. Although many numerical schemes exist for this problem we will focus on hybridizing two approaches with complementary advantages: a volume based method using finite element grids and plane wave basis functions and an overlapping integral equation formulation. Volume methods are derived from a three dimensional discretization of a portion of the exterior domain close to the scatterer and limited by an artificial boundary. If a convenient local boundary condition is used the distance between the scatterer and the artificial boundary must be taken sufficiently large to ensure accuracy. In addition the artificial boundary must be convex. This leads to the expensive discretization of free space. However volume based methods can easily handle non-constant material coefficients, and give rise to sparse matrices. In contrast an integral formulations enables the restriction of the problem to the surface of the scatterer. However, integral techniques require a homogeneous external media, and the evaluation of singular integrals. Both methods are often coupled leading to a hybrid method that inherits the strengths of both component methods.

In this paper, we focus on coupling the Ultra-Weak Variational Formulation (UWVF) and a direct boundary integral representation using a multilevel fast multipole method. The UWVF is a volume based numerical method for solving the time-harmonic Maxwell system on a bounded domain developed by Després and Cessenat $[3,2,12]$. It uses local plane wave solutions on a finite element mesh to approximate impedance traces of the field on the skeleton of the mesh. By varying the number of plane wave basis functions from element to element

${ }^{*}$ The research of PM is partially supported by grant number FA-9550-08-1-0138 from the US AFOSR. This paper is dedicated to Professor George Hsiao on the occasion of his $65^{\text {th }}$ birthday. Professor Hsiao has inspired us by his love for integral equations. 
the UWVF can discretize the electromagnetic field with a coarser volume mesh in comparison to more classical methods low order finite elements or finite differences. It can handle general piecewise constant material properties. However, to approximate scattering on an unbounded domain, the UWVF requires an artificial boundary denoted $\Gamma_{\text {ext }}$ sufficiently far from the scatterer. The original UWVF uses a low order absorbing boundary condition on $\Gamma_{\text {ext }}$ (but see [12] for use of the perfectly matched layer). To overcome the drawback of needing a large distance between the scatterer and a convex artificial boundary, we suggested using an integral representation of the unknown field on $\Gamma_{\text {ext }}$.

In particular we shall use an overlapping scheme. A standard Galerkin volume equation combined with an overlapping integral equation system was first proposed for Maxwell's equations by Hazard and Lenoir [10]. A finite element discretization of the method using edge elements was then analyzed by Hsiao, Monk and Nigam [11]. In particular, Hazard and Lenoir proposed to use an integral representation of the field near the artificial boundary $\Gamma_{\text {ext }}$ computed from unknown field values on a second artificial boundary $\Sigma$ taken closer to the boundary of the scatterer $\Gamma_{\text {int }}$ (see Fig. 1 for a graphic of the relationship of various boundaries). This couples the Cauchy data on $\Sigma$ to the solution on $\Gamma_{\text {ext }}$. The main constraint is that the domain between $\Sigma$ and $\Gamma_{\text {ext }}$ be homogeneous (i.e. the background medium). However the domain within $\Sigma$ can be inhomogeneous. Upon discretization, the well-known integral operators involved in this representation lead to expensive evaluations by direct means (but do not involve singular integrals, or near interaction terms). Indeed the expense and memory needed to compute the linear system resulted in no numerical results being presented in [11]. Clearly the problem can potentially be solved using a fast multipole implementation of the integral equations. Note that for standard edge finite elements this was the approach used by Liu and Jin [13]. However, the UWVF can be more efficient than finite elements at higher wave number, and this motivates our investigations here.

The main aim of coupling the UWVF and a Fast Multipole Method (FMM) is to reduce the overall numerical cost, and we have already introduced the coupled UWVF and FMM in several papers (see for example [4], [14], [9]). In particular in [8], we used a one-level FMM. The numerical results show that the one-level FMM leads to an algorithm with a cost comparable to the UWVF used in its original form. In [7], we gave a thorough study of the complexity of that algorithm using either the one-level or multilevel fast multipole method in different configurations. This paper is devoted to numerical tests of the multilevel version of the algorithm. We shall show that the method can effectively solve several model problems, and give numerical evidence to support the complexity estimates.

The plan of this paper is as follows. The next section gives a brief presentation of the UWVF. In Section 3 , we describe the use of the integral representation within the hybrid UWVF and integral representation (UWVFIR) algorithm, and recall the results on the complexity that were developed in [7]. The last section, which is the focus of this paper, presents numerical results obtained using a multilevel FMM (MLFMM) and demonstrates agreement with the study of complexity. These results show the improved efficiency of the novel algorithm compared to the original UWVF.

\section{Ultra-Weak Variational Formulation}

Suppose we wish to solve the time harmonic Maxwell system in the unbounded exterior of a bounded scatterer (assumed to have connected complement and polyhedral surface $\Gamma_{\text {int }}$ ). Introducing a polyhedral artificial boundary $\Gamma_{\text {ext }}$ containing the scatterer in its interior, we denote by $\Omega$ the bounded annular domain between $\Gamma_{\text {int }}$ and $\Gamma_{\text {ext }}$ (see Fig. 1). We denote by $\nu$ the outward normal to $\Omega$.

To solve the time-harmonic Maxwell equations in a domain $\Omega$ we need to find the electric field $E$ and magnetic field $H$ such that the following equations hold:

$$
\left.\begin{array}{l}
\nabla \wedge E-\imath \omega \mu H=m, \\
\nabla \wedge H+\imath \omega \varepsilon E=j,
\end{array}\right\} \quad \text { in } \Omega
$$

where $m$ and $j$ are given data vector functions specifying the volume sources, $\varepsilon$ and $\mu$ are positive piecewise constant functions of position and $\omega>0$ is the angular frequency of the field. Although not required by the method, we usually assume a source free region and select $m=j=0$ (our numerical test will conform to this).

For the UWVF, it is convenient to specify the boundary condition on $\partial \Omega=\Gamma_{\text {int }} \cup \Gamma_{\text {ext }}$ in the following non standard form $([3])$

$$
-|\sqrt{\varepsilon}| E \wedge \nu+(|\sqrt{\mu}| H \wedge \nu) \wedge \nu=Q(|\sqrt{\varepsilon}| E \wedge \nu+(|\sqrt{\mu}| H \wedge \nu) \wedge \nu)+g,
$$




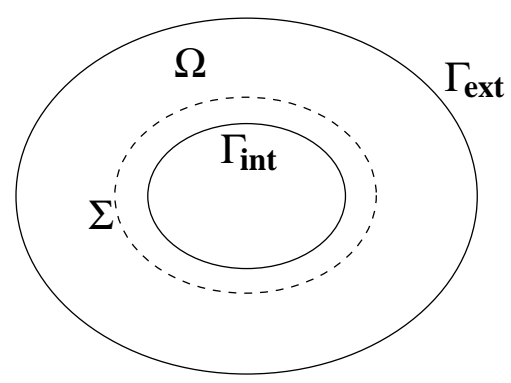

Figure 1: The computational domain $\Omega$ is the region outside the boundary of the impenetrable scatterer $\Gamma_{\text {int }}$ and inside the artificial boundary $\Gamma_{\text {ext }}$. A second artificial boundary $\Sigma$ inside $\Gamma_{\text {ext }}$ and containing the scatterer (both impenetrable and penetrable components) in its interior is used in the integral representation of the field. If the exterior domain is entirely homogeneous and isotropic, we can take $\Sigma=\Gamma_{\text {ext }}$.

where $Q=0$ gives the standard low order absorbing boundary condition on $\Gamma_{\text {ext }}$ and $g$ is computed from the incident wave. Since we model the total field, we choose $g=0$ on $\Gamma_{\text {int }}$ and use $Q$ to set the boundary condition. For example choosing $Q=1$ gives the perfectly conducting boundary condition, while $|Q|<1$ gives an impedance condition. The choice $Q=-1$ gives a magnetic wall condition that is useful for symmetric structures. We choose $Q=0$ on $\Gamma_{\text {ext }}$ and $g$ will be given by an integral operator.

The UWVF is based on the decomposition of the domain $\Omega$ into tetrahedra $\left\{\Omega_{k}\right\}_{k=1, \ldots, K}$ and it computes the impedance trace of the solution on the boundaries of all these tetrahedra (which can then be post processed to give the solution in the entire element). This variational formulation is defined on the Hilbert space $V=$ $\prod_{k=1}^{K} L_{t}^{2}\left(\partial \Omega_{k}\right)$ where $L_{t}^{2}\left(\partial \Omega_{k}\right)$ is the space of square integrable tangential fields on $\partial \Omega_{k}$ the boundary of $\Omega_{k}$. For this space the scalar product is given by

$$
(\mathcal{X}, \mathcal{Y})_{V}=\sum_{k} \int_{\partial \Omega_{k}} \mathcal{X}_{/ \partial \Omega_{k}} \overline{\mathcal{Y} / \partial \Omega_{k}} .
$$

Under the assumption that $\varepsilon$ and $\mu$ are positive constants on each element $\Omega_{k},(E, H)$ is found through the restriction of the field $\left(E_{k}, H_{k}\right)$ to $\partial \Omega_{k}$, where $\left(E_{k}, H_{k}\right)=(E, H) / \Omega_{k}$. The method then solves for an unknown function $\mathcal{X} \in V$, defined element by element by the impedance trace $\mathcal{X}_{/ \partial \Omega_{k}} \in L_{t}^{2}\left(\partial \Omega_{k}\right)$ on $\partial \Omega_{k}$ and

$$
\mathcal{X} / \partial \Omega_{k}=\sqrt{\widetilde{\varepsilon} / \partial \Omega_{k}}\left(E_{k} \wedge \nu_{k}\right)+\sqrt{\widetilde{\mu} / \partial \Omega_{k}}\left(\left(H_{k} \wedge \nu_{k}\right) \wedge \nu_{k}\right) .
$$

where $\widetilde{\varepsilon} / \partial \Omega_{k}$ and $\widetilde{\mu} / \partial \Omega_{k}$ are quantities defined by the values of $\varepsilon$ and $\mu$ on each side of $\partial \Omega_{k}$ (see [8] for details), and $\nu_{k}$ is the exterior normal to $\partial \Omega_{k}$.

The UWVF involves two operators $\Pi$ and $F$ defined in $[3,2]$. The operator $\Pi: V \rightarrow V$ switches boundary traces across faces shared by two tetrahedra and involves the boundary condition (2) through the function $Q$ on faces which contribute to the boundaries $\Gamma_{\text {ext }}$ and $\Gamma_{\text {int }}$. More precisely if elements $\Omega_{j}$ and $\Omega_{k}$ meet at a face $f$ then

$$
\left.\Pi \mathcal{X}_{j}\right|_{f_{j, k}}=\left.\mathcal{X}_{k}\right|_{f_{j, k}}
$$

and on a boundary face of an element $\Omega_{k},\left.\Pi \mathcal{X}_{k}\right|_{\partial \Omega}=\left.Q \mathcal{X}_{k}\right|_{\partial \Omega}$. The operator $F: V \rightarrow V$ is a local operator that links the outgoing and incoming impedance traces of the degrees of freedom on the interfaces between tetrahedra. If $(u, v)$ satisfy the Maxwell system on $\Omega_{K}$ and $\mathcal{X}_{k}=\sqrt{\widetilde{\varepsilon} / \partial \Omega_{k}}\left(u \wedge \nu_{k}\right)+\sqrt{\widetilde{\mu} / \partial \Omega_{k}}\left(\left(v \wedge \nu_{k}\right) \wedge \nu_{k}\right)$. then $F\left(\mathcal{X}_{k}\right)=\sqrt{\widetilde{\varepsilon} / \partial \Omega_{k}}\left(u \wedge \nu_{k}\right)-\sqrt{\widetilde{\mu} / \partial \Omega_{k}}\left(\left(v \wedge \nu_{k}\right) \wedge \nu_{k}\right)$.

The UWVF of Maxwell's equations is as follows [3, 2]: Find $\mathcal{X} \in V$ such that

$$
(\mathcal{X}, \mathcal{Y})_{V}-(\Pi \mathcal{X}, F \mathcal{Y})_{V}=(\widetilde{b}, \mathcal{Y})_{V} \quad \text { for all } \mathcal{Y} \in V
$$

for all $\mathcal{Y} \in V$ where $\tilde{g}$ is the extension by zero of $g$ to a function in $V$.

Thus by taking a finite dimensional subspace $V_{h} \subset V$ and using basis functions $Z_{i}, i \in J$ for $V_{h}$, a Galerkin discretization of the formulation (4) leads to problem of finding $\mathcal{X}_{h}=\sum_{i \in J} X_{i} Z_{i} \in V_{h}$ such that $\left(\mathcal{X}_{h}, \mathcal{Y}_{h}\right)_{V}-$ $\left(\Pi \mathcal{X}_{h}, F \mathcal{Y}_{h}\right)_{V}=\left(\widetilde{g}, \mathcal{Y}_{h}\right)_{V}$ for all $\mathcal{Y}_{h} \in V_{h}$. 
Equivalently, in matrix/vector form, we seek to compute $X=\left[X_{1}, \cdots, X_{\operatorname{card}(J)}\right]^{T}$ such that

$$
(D-C) X=b,
$$

where $D$ is the matrix with $(i, j)$ th entry $\left(Z_{j}, Z_{i}\right)_{V}$ and $C$ has $(i, j)$ th entry given by $\left(\Pi Z_{j}, F Z_{i}\right)_{V}$. The data vector $b$ is derived from the right hand side above in the same way.

As usual for the UWVF, to facilitate calculating the action of $F$, on each element $\Omega_{k}$ we use a basis generated by taking the impedance trace of $p_{k}$ plane waves satisfying the adjoint Maxwell system on $\Omega_{k}\left(p_{k} / 2\right.$ directions with two polarizations for each direction). In particular to discretize the problem, we follow [3] and use boundary functions given by

$$
\mathcal{Y}_{/ \partial \Omega_{k}}=\sqrt{\widetilde{\varepsilon}_{/ \partial \Omega_{k}}}\left(E_{k}^{\prime} \wedge \nu_{k}\right)+\sqrt{\widetilde{\mu}_{/ \partial \Omega_{k}}}\left(\left(H_{k}^{\prime} \wedge \nu_{k}\right) \wedge \nu_{k}\right)
$$

where the fields $\left(E_{k}^{\prime}, H_{k}^{\prime}\right)$ are taken from the span of a set of $p_{k}$ plane waves that satisfy the adjoint Maxwell problem

$$
\left\{\begin{array}{l}
\nabla \wedge E_{k}^{\prime}-\imath \omega \overline{\mu_{\Omega_{k}}} H_{k}^{\prime}=0 \text { in } \Omega_{k} \\
\nabla \wedge H_{k}^{\prime}+\imath \omega \overline{\varepsilon_{\Omega_{k}}} E_{k}^{\prime}=0 \text { in } \Omega_{k}
\end{array}\right.
$$

In (4), $\widetilde{b} \in V$ is derived from the right hand side of (1) and from $g$ given in (2). At least six plane waves (and usually more) are used per element, counting polarizations.

The UWVF then leads to a sparse square system of size $\left(\sum_{k=1}^{K} p_{k}\right)$. The number of plane waves $p_{k}$ is chosen depending on the local wavelength and diameter of the element (see [12]).

Compared to more classical volume methods, the UWVF enables one to reduce the number of elements in the mesh. The complexity of the method is then linked to the number of elements in the mesh and the number of basis functions per element. For concreteness, suppose the electromagnetic parameters of the domain are constant and define the wave number $\kappa=\omega \sqrt{\varepsilon \mu}$. Before a closer consideration of the complexity, we need to introduce another parameter: $K_{0}$ denotes the average number of tetrahedra taken in one dimension so that $K \sim K_{0}^{3}$. As a volume method, the UWVF method leads to a sparse system: The number of degrees of freedom is of order $K_{0}^{3} p$ and the complexity of the algorithm is $\mathcal{O}\left(K_{0}^{3} p^{2}\right)$ where $p$ denotes the average number of basis functions per tetrahedra which typically satisfies $K_{0} p \sim \kappa[12]$.

\section{Use of an Integral Representation within the UWVF}

To simplify the presentation we shall suppose that $\varepsilon=\mu=1$ so that the exterior domain is entirely homogeneous and we use the perfectly conducting boundary condition on $\Gamma_{\text {int }}$ (i.e. the scatterer is not penetrable and the exterior medium is homogeneous). In this case we may take $\Sigma=\Gamma_{\text {int }}$ (extensions to more general domains in which $\Sigma \neq \Gamma_{\text {int }}$ is then easy to understand). In this case the artificial boundary $\Gamma_{\text {ext }}$ can then be taken very close to the boundary of the obstacle. Following Hazard and Lenoir [10], the hybrid scheme consists in replacing the low order absorbing boundary condition $-E \wedge \nu+(H \wedge \nu) \wedge \nu=-E_{0} \wedge \nu+\left(H_{0} \wedge \nu\right) \wedge \nu$ on $\Gamma_{\text {ext }}$ by the boundary condition

$$
-E \wedge \nu+(H \wedge \nu) \wedge \nu=-E^{s} \wedge \nu+\left(H^{s} \wedge \nu\right) \wedge \nu-E_{0} \wedge \nu+\left(H_{0} \wedge \nu\right) \wedge \nu,
$$

where $\left(E^{s}, H^{s}\right)$ are given by the Stratton-Chu formula ([5]) in terms of $\nu \times H$ and $\nu \times E$ on $\Sigma$ (i.e. under our assumptions on $\left.\Gamma_{\text {int }}\right)$ via

$$
\begin{aligned}
E^{s}(x)= & \nabla_{x} \wedge \int_{\Gamma_{\mathrm{int}}} G(x, y) \nu_{\Sigma}(y) \wedge E(y) d \gamma(y) \\
& -\frac{1}{\imath \omega} \nabla_{x} \wedge \nabla_{x} \wedge \int_{\Gamma_{\mathrm{int}}} G(x, y) \nu_{\Sigma}(y) \wedge H(y) d \gamma(y), \\
H^{s}(x)= & \nabla_{x} \wedge \int_{\Gamma_{\mathrm{int}}} G(x, y) \nu_{\Sigma}(y) \wedge H(y) d \gamma(y) \\
& +\frac{1}{\imath \omega} \nabla_{x} \wedge \nabla_{x} \wedge \int_{\Gamma_{\mathrm{int}}} G(x, y) \nu_{\Sigma}(y) \wedge E(y) d \gamma(y),
\end{aligned}
$$


where $\nu_{\Sigma}$ is the exterior normal to the surface $\Sigma=\Gamma_{\text {int }}$ and $G(x, y)=\exp (\iota \kappa|x-y|) /(4 \pi|x-y|)$ is the fundamental solution for the Helmholtz equation. Thanks to the structure of the unknowns of the UWVF, as shown in [8], the fields in the integrands above can be computed directly from the degrees of freedom of the UWVF (3) taking into account the convention for the direction of normals, and the boundary condition on $\Gamma_{\text {int }}$ (2) where $g=0$.

The system (5) becomes $(D-C-\widetilde{C}) X=b$ where $\widetilde{C}$ couples the degrees of freedom on $\Gamma_{\text {int }}$ (more generally $\Sigma)$ and $\Gamma_{\text {ext }}$. The matrix $\widetilde{C}$ can be split into different discrete integral operators $\widetilde{C}_{i}, i=1, \ldots, 4$ of the form

$$
\left(\widetilde{C}_{i} \mathcal{X}_{h}\right)_{k l}=\int_{\Sigma_{k k}^{\mathrm{ext}}} c_{k} S_{i}\left(\mathcal{X}_{h}\right) \cdot \overline{F \mathcal{Y}_{k l}} d \gamma_{\mathrm{ext}}
$$

where

- $\Sigma_{k k}^{\text {ext }}$ is the face on $\Gamma_{\text {ext }}$ of a tetrahedron intersects the exterior boundary,

- $c_{k}$ depends only on $\varepsilon$ and $\mu$ on $\Sigma_{k k}^{\text {ext }}$,

- $F$ is the local operator introduced in (4),

- $S_{i}$ is a global operator which comes from the right hand side of (6)-(7), for instance

$$
\left(S_{1}(\mathcal{X})\right)(x)=\left(-\int_{\Gamma_{\text {int }}} f_{Q}(y) \nabla_{y} G(x, y) \wedge \mathcal{X}(y) d \gamma(y)\right) \wedge \nu(x)
$$

where $f_{Q}$ is a function involving $Q$ and $\varepsilon$. These integral operators can be evaluated by the FMM.

In this paper, the solution of the new system $(D-C-\widetilde{C}) X=b$ is obtained by the same method (BiCGStab) as used for the classical UWVF system $(D-C) X=b$, considering $C+\widetilde{C}$ as a small perturbation of $C$. It is important to note that we never compute $\tilde{C}$ explicitly, but evaluate its action as needed by the FMM.

The integral representation aims to reduce the distance of the absorbing boundary from the scatterer to a number of elements independent of $\kappa$. We then have a number of elements in the mesh of order $K_{0}^{2}$. This reduces the complexity related to the volume calculation. The FMM is used to control the cost of the integral calculation related to the integral operators which give rise to large dense blocks in the discrete system.

A rigorous expression of the algorithm complexity involves several parameters: the size of the mesh $K \sim$ $K_{0}^{3}$ or $K_{0}^{2}$, the average number of basis functions per tetrahedron $p$, the wave number $\kappa$, with the correlation $K_{0} p \sim \kappa$. Moreover, the integral representation involves oscillating functions such as the Green function, and the exterior normal to the boundaries. Evaluation of these operators requires an accurate model of the boundary which is assured by our assumption of a polyhedral domain. For more general surfaces it a possible modification of our algorithm would be to use a coarse volume mesh for the UWVF and a finer surface mesh for the integral calculations (the mesh used for the resolution of classical integral equations); this follows the double-mesh concept introduced by Zhou et al. [1] and used in [6]. Furthermore, note that the FMM used here does not involve very close interactions. Indeed, the integral operators link points on one boundary to points on another boundary so the source points are not in the same domain as the target points. All these considerations lead to different algorithms with different complexities. In Tab. 1, we give the results developed in [7] which suggest that the UWVF with MLFMM and no close interactions should have attractive work estimates.

\section{$4 \quad$ Numerical Results}

In this section, we give numerical results with the new algorithm which couples the UWVF and the integral representation using a MLFMM. Our first results are for the problem of approximating scattering by a perfectly conducting unit sphere for $\left.\Gamma_{\text {int }}\right)$ with the wave numbers $\kappa=3 \mathrm{~m}^{-1}, 4 \mathrm{~m}^{-1}, 6 \mathrm{~m}^{-1}, 10 \mathrm{~m}^{-1}, 15 \mathrm{~m}^{-1}$. The wavelength is then $\lambda=2 \pi / 3 \approx 2.09 \mathrm{~m}, \pi / 2 \approx 1.6 \mathrm{~m}, \pi / 3 \approx 1.05 \mathrm{~m}, \pi / 5 \approx 0.63 \mathrm{~m}, 2 \pi / 15 \approx 0.42 \mathrm{~m}$. This very simple example has the advantage that a Mie series solution is available for comparison.

We also note that the method easily extends to disconnected scatterers. If the scatterers are close together (closer than approximately 4 elements in the mesh), we need to surround the scatterers by a single auxilary boundary. In this case multiple scattering is taken into account by the UWVF. If the scatterers are sufficiently 
Table 1: Complexity estimates for the various versions of the UWVF + IR

\begin{tabular}{|l|c|c|}
\hline \multicolumn{1}{|c|}{ Method } & Number of elements & Cost of the solution \\
\hline UWVF & $K_{0}^{3} \sim(\kappa / p)^{3}$ & $K_{0}^{3} p^{2} \sim K_{0} \kappa^{2}$ \\
\hline UWVF + IR & $K_{0}^{2} \sim \kappa^{2}$ & $K_{0}^{2} p^{2}+K_{0}^{4} p^{2} \sim \kappa^{2}+\kappa^{4}$ \\
\hline UWVF + IR + 1-level FMM & $K_{0}^{2} \sim \kappa^{2}$ & $K_{0}^{2} p^{2}+K_{0}^{3} p \sim \kappa^{2}+\kappa^{3}$ \\
\hline UWVF + IR + multilevel FMM & $K_{0}^{2} \sim \kappa^{2}$ & $K_{0}^{2} p^{2}+K_{0}^{2} \ln ^{2}\left(K_{0}\right) p \sim \kappa^{2}+\kappa^{2} \ln ^{2} \kappa$ \\
\hline $\begin{array}{l}\text { UWVF + IR + multilevel FMM } \\
\text { with double mesh }\end{array}$ & $K_{0}^{2} \sim(\kappa / p)^{2}$ & $K_{0}^{2} p^{2}+\kappa^{2} \ln ^{2}(\kappa) p \sim \kappa^{2}+\kappa^{2} \ln ^{2}(\kappa) p$ \\
\hline $\begin{array}{l}\text { UWVF + IR + 1-level FMM } \\
\text { without close interaction }\end{array}$ & $K_{0}^{5 / 2} \sim \kappa^{5 / 2}$ & $K_{0}^{5 / 2} p^{2}+K_{0}^{3} p \sim \kappa^{2}+\kappa^{3}$ \\
\hline $\begin{array}{l}\text { UWVF + IR + multilevel FMM } \\
\text { without close interaction }\end{array}$ & $K_{0}^{2} \sim \kappa^{2}$ & $K_{0}^{2} p^{2}+K_{0}^{2} \ln ^{2}\left(K_{0}\right) p \sim \kappa^{2}+\kappa^{2} \ln ^{2} \kappa$ \\
\hline
\end{tabular}

Table 2: The meshes for the unit sphere.

\begin{tabular}{|l|c|c|c|c|c|c|c|}
\hline Name & $\mathrm{S} 400$ & $\mathrm{~S} 200$ & $\mathrm{~S} 040$ & $\mathrm{~S} 025$ & $\mathrm{~S} 017$ & S010 & S007 \\
\hline$\kappa$ & 4 & 4 & 3 & 4 & 6 & 10 & 15 \\
\hline Radius of $\Gamma_{\text {ext }}$ in $\mathrm{m}$ & 5 & 3 & 1.40 & 1.25 & 1.17 & 1.10 & 1.07 \\
\hline Distance between $\Gamma_{\text {int }}$ and $\Gamma_{\text {ext }}$ & $\approx 2.5 \lambda$ & $\approx 1.3 \lambda$ & $\approx \lambda / 7.5$ & $\approx \lambda / 6.3$ & $\approx \lambda / 6.4$ & $\approx \lambda / 6.3$ & $\approx \lambda / 6.6$ \\
\hline Number of tetrahedra & 16179 & 14526 & 5822 & 11008 & 8449 & 22630 & 44459 \\
\hline $\begin{array}{l}\text { Number of basis functions } \\
\text { per tetrahedron }\end{array}$ & 8 to 128 & 8 to 72 & 10 to 22 & 10 to 24 & 16 to 30 & 16 to 28 & 14 to 30 \\
\hline Number of DoF & 880200 & 508450 & 101814 & 178146 & 183108 & 480226 & 989238 \\
\hline
\end{tabular}

separated, we can use a thin layer of elements around each scatterer. In this case multiple scattering is taken into account via the fast multipole method and hence dispersion error is reduced. We have not yet found it necessary to precondition in a special way in these cases.

Results from three codes are presented: the classical UWVF with a Silver-Müller type low order auxilliary boundary condition of order 0 (i.e the standard UWVF with the boundary condition described earlier setting $Q=0$ ), the code UWVF+FMM using a 1-level FMM and the code UWVF+MLFMM using a multi level FMM. All the resuts in this paper were obtained on an Apple Mac Pro with $2 \times 3 \mathrm{GHz}$ quad-core Xeon processors using 16 Gb RAM.

\subsection{The unit sphere}

For approximating scattering by the unit sphere, the exterior boundary $\Gamma_{\text {ext }}$ is taken to be a concentric sphere. Obviously these are not polyhedral domains, but can be approximated using a sufficiently fine mesh near the boundary. We have experimented with several exterior boundaries giving rise to different meshes as defined in [8]. Table 2 describes those used in this paper. The names "Sxxx" denote the different meshes, where "xxx" denotes one of the numbers 400, 200, 100, 075, 050, 025, 040, 017. This number gives the distance between $\Gamma_{\text {int }}$ and $\Gamma_{\text {ext }}$ in centimeters. For $\kappa=4$ the distance from the perfect conductor to the artificial boundary ranges from $0.16 \lambda$ (S025) to $2.5 \lambda$ (S400) where $\lambda=2 \pi / \kappa$ is the wavelength.

All the meshes have been generated using COMSOL Multiphysics version 3.5a. The meshes S400 and S200 are appropriate for a classical use of the UWVF. The meshes S025, S040, S017, S010 and S007 have been generated optimizing the ratio between the average edge-length $h$ and the wavelength $\lambda$ to $h \approx \lambda / 5$. These meshes are quite uniform (the $S 400$ and $S 200$ meshes are graded to give larger elements away from the scatterer). The large number of tetrahedra in S025 might appear to be a disadvantage for the UWVF+MLFMM method (the number of tetrahedra is comparable to S400). However the number of Degrees of Freedom (DoF) is much less than for S400 because fewer plane waves are used per element due to the smaller size of elements as shown in the bottom row of Table 2 .

We use the radar cross section (RCS) as a measure of the efficiency of the method since the RCS is often 

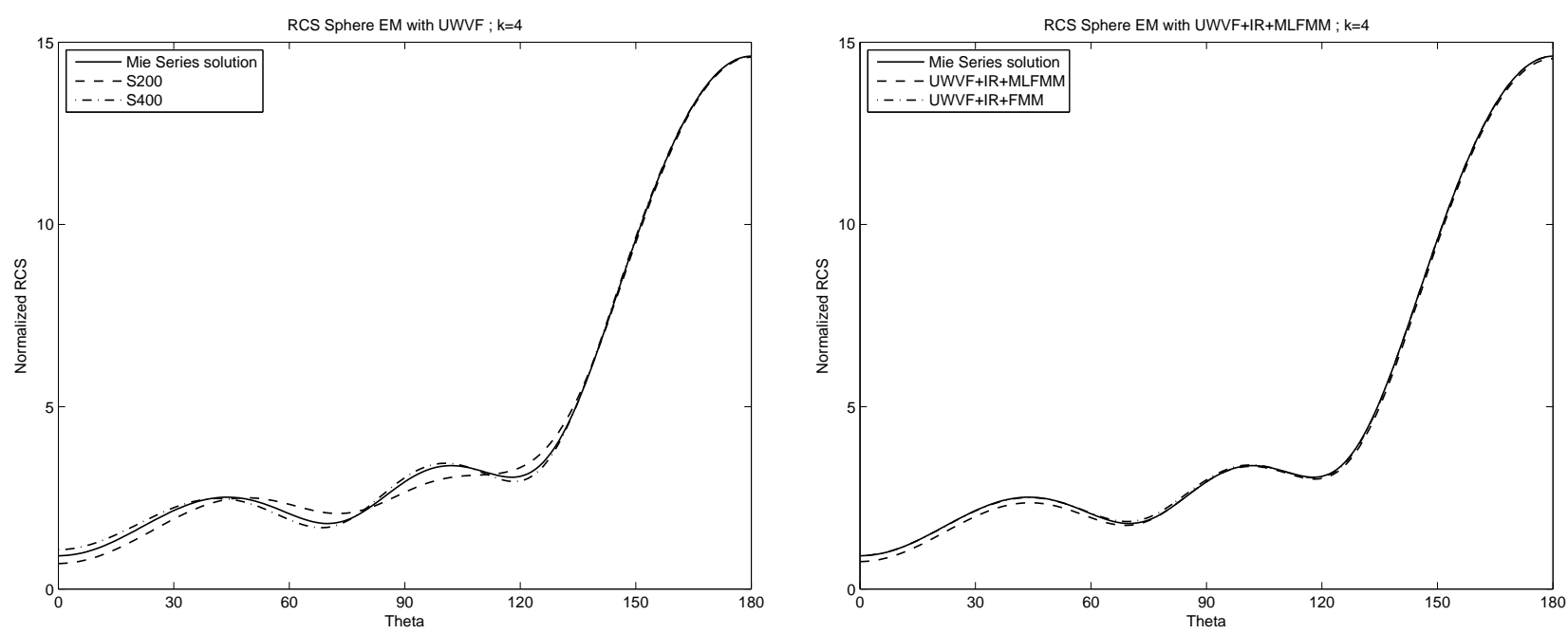

Figure 2: The TE-polarized RCS as a function of polar coordinate $\theta$ computed using the three codes in the study, with $\kappa=4$. Left: results for the classical UWVF code with meshes S400 and S200. Right: results for the codes UWVF+FMM and UWVF+MLFMM with the mesh S025.

the desired output of the code. The RCS is obtained from the far field pattern of the dielectric field denoted $E_{\infty}$ as follows:

$$
\mathrm{RCS}=10 \log _{10}\left(2 \pi \kappa\left\|E_{\infty}\right\|^{2}\right) .
$$

We start by comparing the classical UWVF, the UWVF-FMM and UWVF-MLFMM at constant wave number $\kappa=4$. Figure 2 compares the results of our codes as a function of angle with the exact Mie series solution for TE polarization, and Fig. 3 shows results for TM polarization, but only for UWVF-MLFMM. As is well known, the classical UWVF code requires a large distance between $\Gamma_{\text {int }}$ and $\Gamma_{\text {ext }}$ to obtain an accurate solution due to its low order absorbing boundary condition. Figure 2 clearly shows the impact of the integral representation on the accuracy of the results for the case $\kappa=4$. The code UWVF+FMM gives results which fit with the Mie series solution, even with the thin mesh S025. The results obtained with the code UWVF+MLFMM are comparable to the those of the code UWVF+FMM, indicating that use of the multilevel scheme does not degrade the FMM accuracy.

CPU-time, memory requirements and error measures are given in the case of the TE polarization in Table 3. The results are given for the meshes S400 and S200 using the classical UWVF code and for the mesh S025 using the codes UWVF+FMM and UWVF+MLFMM. In the table we use the following notation (units are seconds and Giga-bytes):

- CPU time:

T00 $=$ precalculation of the matrices $C$ and $D$.

T0c $($ resp. T0f $)=$ precalculation of the close (resp. far) interactions related to $\widetilde{C}$.

$\mathrm{TD}\left(\right.$ resp. TC) = one multiplication by $D^{-1}$ (resp. $\left.C\right)$ (average).

TCc (resp. TCf) = one multiplication by $\widetilde{C}$ close (resp. far) (average).

$\mathrm{Tcg}=$ Total CPU time for the solution of the system.

Ttot $=$ Total CPU time required by the code.

- Nit $=$ Number of iterations for the bi-conjuguate gradient.

- mem = Memory required by the code.

- Rel Err 2 = Relative quadratic error on RCS. 


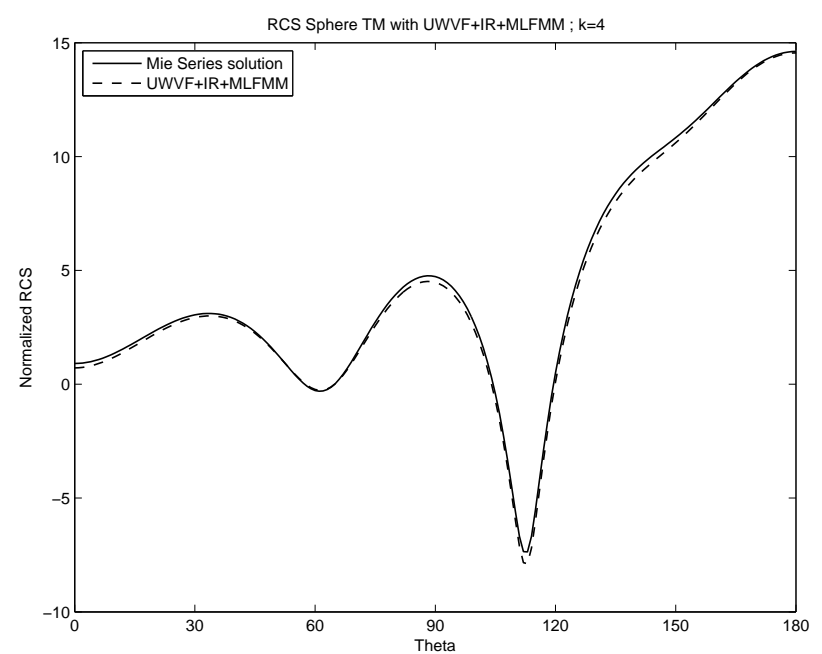

Figure 3: The TM-polarized RCS against polar angle $\theta$ computed using the code UWVF+MLFMM (dashed line) with the mesh S025 and the exact solution (solid line). Here $\kappa=4$.

Table 3: Computational costs comparisons between the UWVF (S400; S200), UWVF+FMM (S025 - F) and UWVF+MLFMM ( S025 - M) for scattering by a unit sphere at constant $\kappa$. For more details of the meshes see Table 2. The RCS error is computed via the Mie series.

\begin{tabular}{|c||c||c|c|c||c|c|c|c|c|c|}
\hline Case & $\kappa$ & T00 & T0c & T0f & TD & TC & TCc & TCf & Tcg & Nit \\
\hline \hline S400 & 4 & 326 & - & - & 1.2 & 7.6 & - & - & 1399 & 156 \\
\hline S200 & 4 & 73.7 & - & - & 0.4 & 1.99 & - & - & 388 & 155 \\
\hline S025 - F & 4 & 34 & 138.6 & 4.3 & 0.1 & 0.2 & 1 & 3.5 & 656 & 135 \\
\hline S025 - M & 4 & 10.4 & 52 & 2.98 & 0.11 & 0.3 & 0.36 & 4.5 & 733 & 139 \\
\hline
\end{tabular}

\begin{tabular}{|c|c|c|c|c|c|c|}
\hline Case & $\kappa$ & Ttot & mem & Rel Err 2 & Rel Err $\infty$ & RMS error \\
\hline$\overline{\overline{\text { S400 }}}$ & 4 & $\bar{~} 1725$ & $\overline{\overline{6.2}}$ & $\bar{c} 1.510^{-2}$ & $\bar{~} 1.110^{-2}$ & 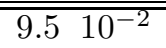 \\
\hline$\overline{\mathrm{S} 200}$ & 4 & 462 & 1.8 & $3.210^{-2}$ & $2.510^{-2}$ & $210^{-1}$ \\
\hline $\mathrm{S} 025-\mathrm{F}$ & 4 & 838 & 2.8 & $4.610^{-3}$ & $5.110^{-3}$ & $2.810^{-2}$ \\
\hline S025 - M & 4 & 798 & 1.6 & $1.710^{-2}$ & $1.110^{-2}$ & $1.110^{-1}$ \\
\hline
\end{tabular}

- Rel Err $\infty=$ Relative infinity error on RCS.

- RMS error $=$ Root mean square error on the scattering amplitude. Like in [15],

$$
\text { RMS error }=\sqrt{\left(\frac{1}{n}\right) \sum_{i=1}^{n}(R C S(i)-\operatorname{Exact}-R C S(i))^{2}}
$$

is used as a classical RCS error ([15]).

The RCS curve obtained with S200 using the UWVF code could be acceptable, however its accuracy is quite poor in comparison with the one obtained with S025 using the codes UWVF+FMM and UWVF+MLFMM hence we should compare results for S400 and S025 in Table 3. That case shows that the UWVF-MLFMM code reduces computer time by approximately $50 \%$ and the memory needed by almost $75 \%$. for roughly the same RMS error.

Next we investigate the $\kappa$ dependence of the method to determine how well Table 1 reflects the practical work needed by the method. Of course, as $\kappa$ increases the method needs to add degrees of freedom to maintain accuracy. Moreover our crude geometry approximation requires a finer mesh as $\kappa$ increases. Hence we use 

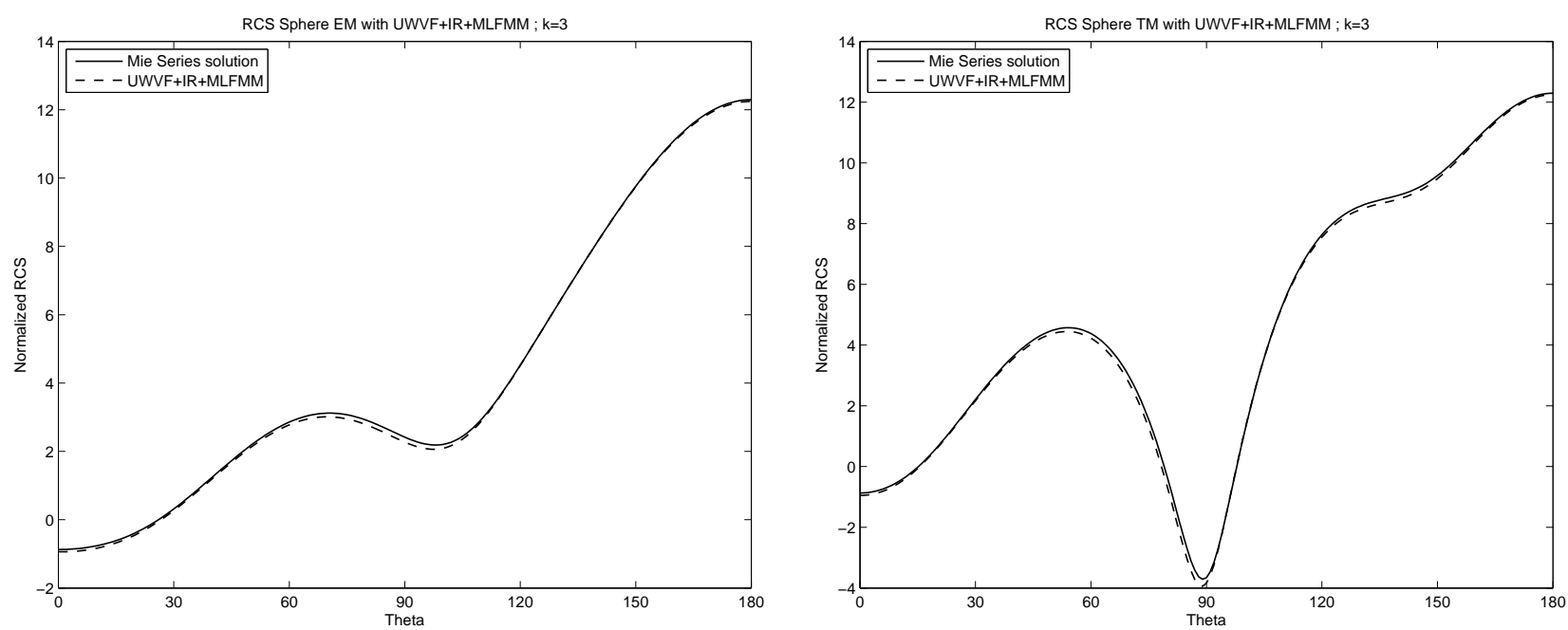

Figure 4: The RCS computed using UWVF+MLFMM with the mesh S040, with $\kappa=3$. Left: TE-polarized RCS. Right: TM-polarized RCS.
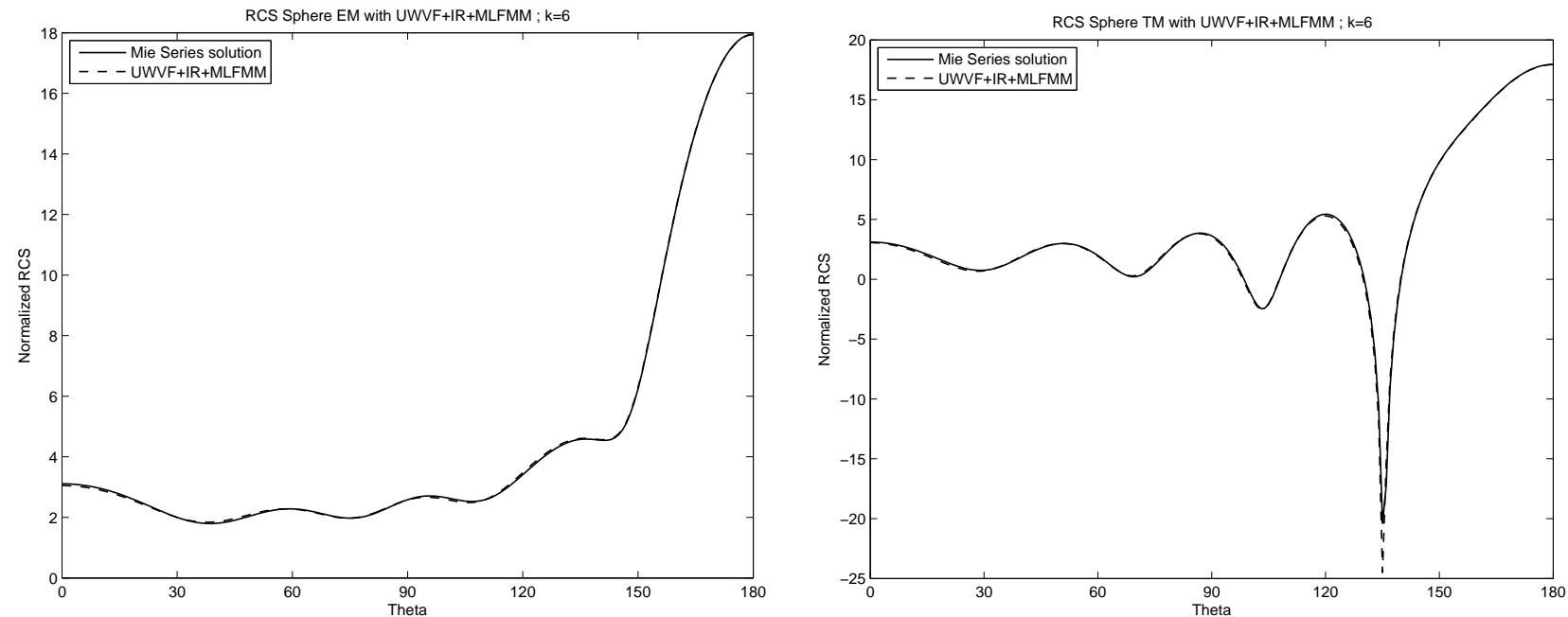

Figure 5: The RCS computed using UWVF+MLFMM with the mesh S017, with $\kappa=6$. Left: TE-polarized RCS. Right: TM-polarized RCS. 

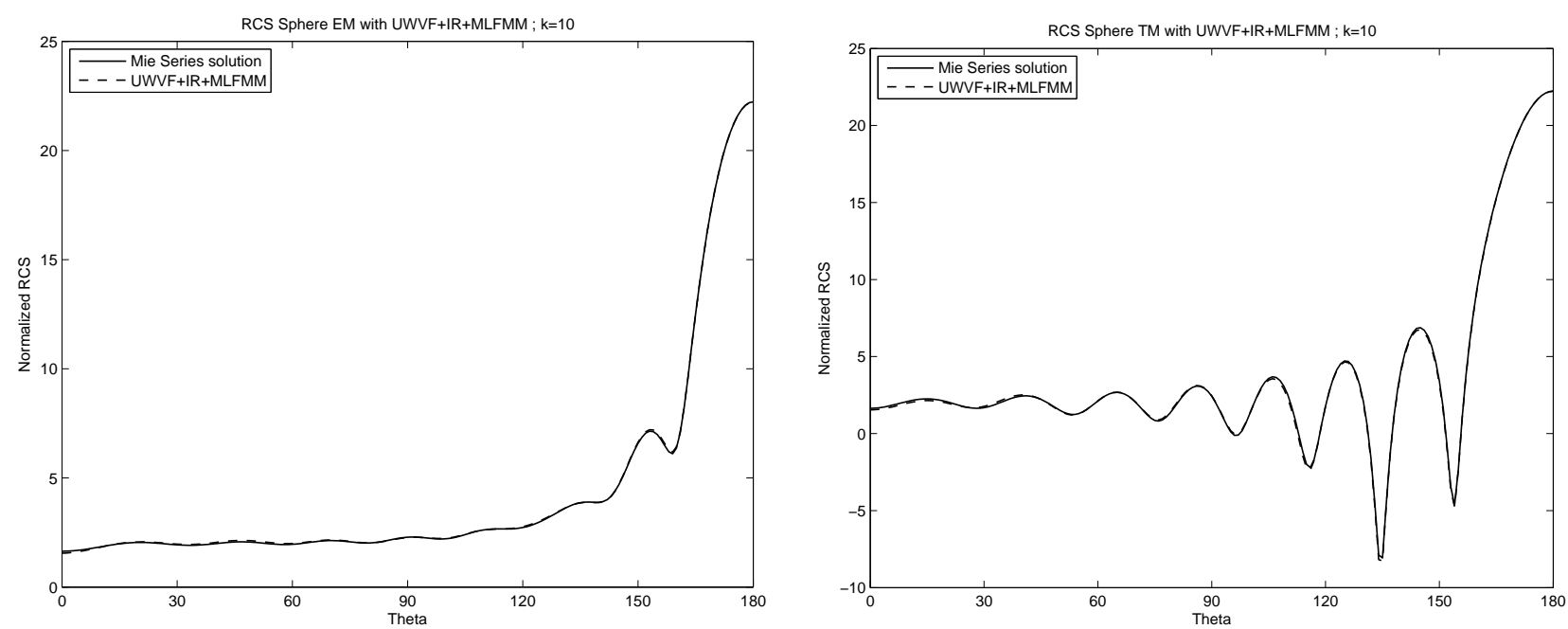

Figure 6: The RCS computed using UWVF+MLFMM with the mesh S010, with $\kappa=10$. Left: TE-polarized RCS. Right: TM-polarized RCS.
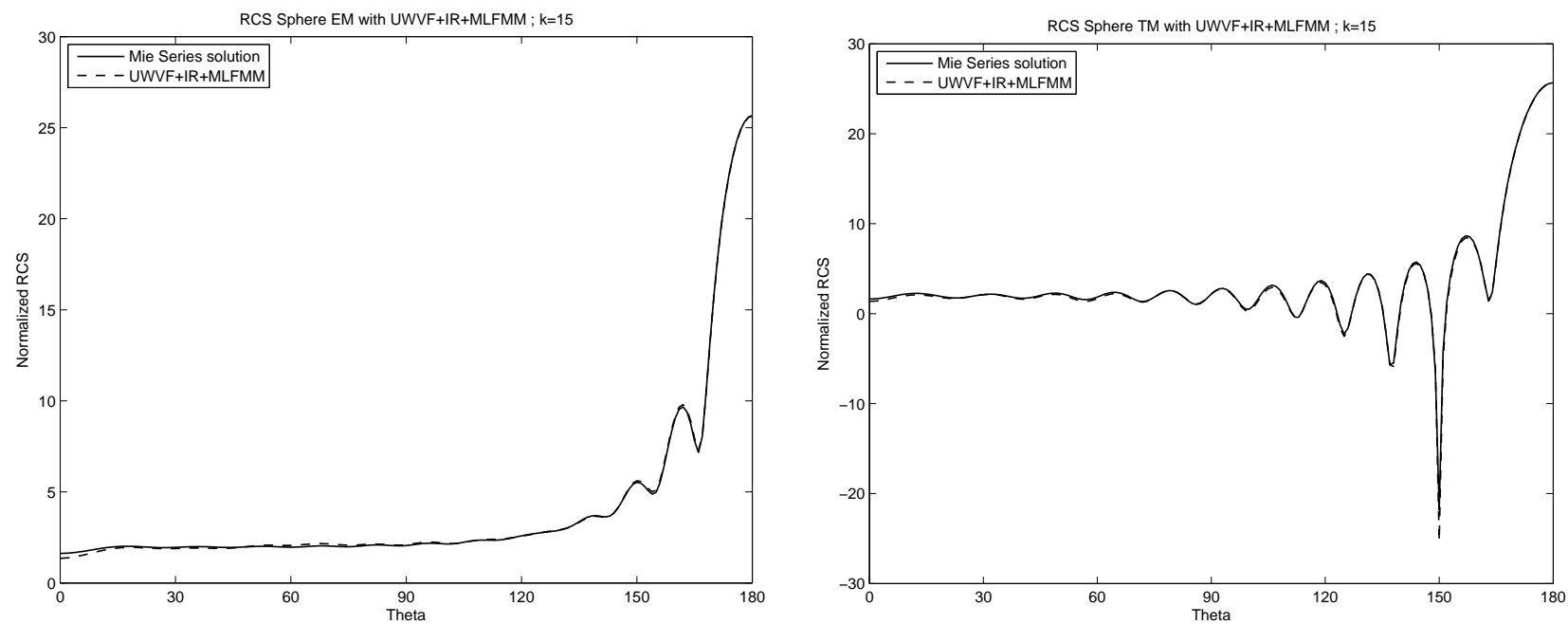

Figure 7: The RCS computed using UWVF+MLFMM with the mesh S007, with $\kappa=15$. Left: TE-polarized RCS. Right: TM-polarized RCS. 
Table 4: Computational costs comparison for UWVF+MLFMM (S040 ; S025 - M ; S017 ; S010 ; S007) as $\kappa$ changes. For more details of the meshes see Table 2. All results are for the unit sphere and the RCS error is computed via the Mie series.

\begin{tabular}{|c||c||c|c|c||c|c|c|c|c|c|}
\hline Case & $\kappa$ & T00 & T0c & T0f & TD & TC & TCc & TCf & Tcg & Nit \\
\hline \hline S040 & 3 & 6.81 & 80 & 2.7 & 0.06 & 0.18 & 0.5 & 3 & 469 & 125 \\
\hline S025 - M & 4 & 10.4 & 52 & 2.98 & 0.11 & 0.3 & 0.36 & 4.5 & 733 & 139 \\
\hline S017 & 6 & 13.5 & 141 & 5.7 & 0.1 & 0.4 & 0.95 & 7.9 & 894 & 95 \\
\hline S010 & 10 & 35 & 467 & 15 & 0.3 & 1 & 3 & 19.6 & 2252 & 93 \\
\hline S007 & 15 & 76 & 921 & 66 & 1.3 & 4.5 & 16.7 & 61.5 & 8880 & 105 \\
\hline
\end{tabular}

\begin{tabular}{|c|c|c|c|c|c|c|}
\hline Case & $\kappa$ & Ttot & mem & Rel Err 2 & Rel Err $\infty$ & RMS error \\
\hline$\overline{\overline{\text { S040 }}}$ & & $\overline{558}$ & 1.8 & 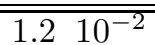 & $\overline{1.210^{-2}}$ & $\overline{77.310^{-2}}$ \\
\hline S025. & & 79 & 1.8 & $1.7 \quad 10^{-2}$ & 1.110 & $1.1 \quad 10^{-1}$ \\
\hline$\overline{\mathrm{S} 017}$ & & $\overline{054}$ & 2.99 & $6.410^{-}$ & $4.5 \quad 10^{-}$ & $4.1 \quad 10^{-2}$ \\
\hline S010 & 10 & 2770 & 8.38 & $5.510^{-3}$ & $4.110^{-3}$ & $\begin{array}{ll}3.6 & 10^{-2}\end{array}$ \\
\hline S007 & 15 & 9944 & 17 & $1.3 \quad 10^{-2}$ & $1.110^{-2}$ & $8.5 \quad 10^{-2}$ \\
\hline
\end{tabular}
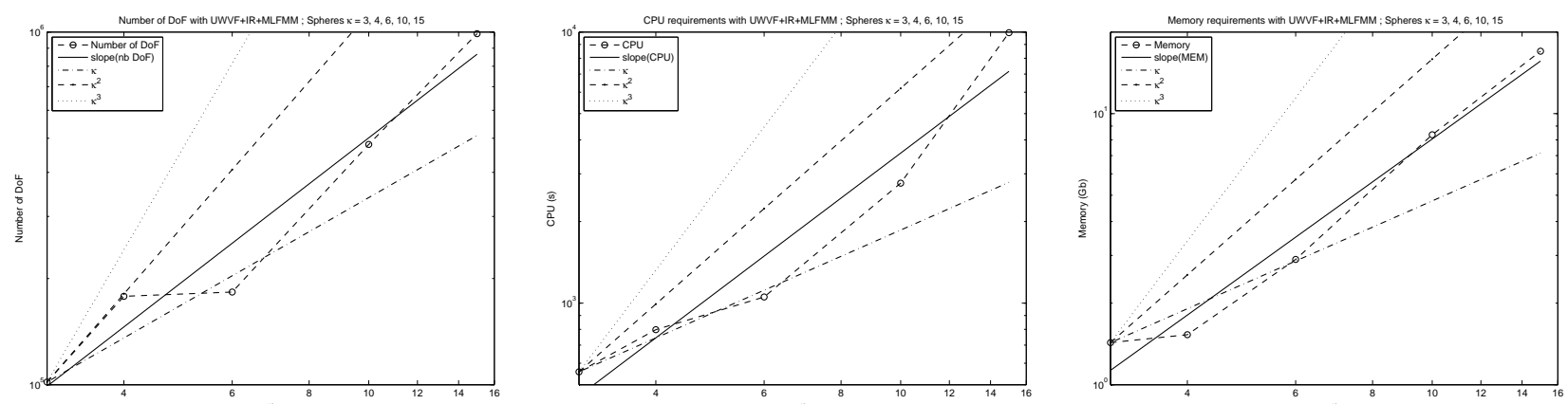

Figure 8: Number of degrees of freedom (left), CPU-time (middle) and memory requirements (right) with respect to the wave number $\kappa$, using a log-log scale. We show the data from Table 4 marked with a symbol in each case. The best fit line is shown as a solid line, and lines corresponding to $O(\kappa), O\left(\kappa^{2}\right)$ and $O\left(\kappa^{3}\right)$ for comparison.

meshes S040, S035, S017, S010 and S007. Figures 3, 4 and 5 show the angular dependence of the RCS from our UWVF-MLFMM code compared to the exact Mie series.

Memory, CPU and time requirements, together with quantitative error results are shown in Table 4. We have tried to keep the RMS error roughly constant to enable comparison. We then plot the resulting number of degrees of freedom, CPU and memory requirements against $\kappa$ in Fig.8. We also consider reference lines to enable comparison with results predicted in Table 4 . These curves support the theoretical results on the complexity.

\section{$4.2 \quad$ Multiple scatterers}

In this subsection, we show results obtained on two different multiple-scatterers examples with the wave number $\kappa=4$. The first result (Figure 10) is obtained with UWVF+MLFMM on a scatterer consisting of two closely separated spheres using a mesh named $2 \mathrm{~S} 025$. This scatterer consists of two unit spheres centered at $( \pm 1.125,0,0)$, and compared to UWVF with $2 \mathrm{~S} 200$ and $2 \mathrm{~S} 400$ where the name of the meshes were chosen as before. For the different cases, the artificial boundary is taken around the two spheres such that the domain is as in the two leftmost panels of Figure 9. Because the scatterers are close together in comparison to our meshing constraints (i.e. closer than 4 elements apart), we need to use a single mesh surrounding both scatterers. For the meshes 2S200 and 2S400 that surround two balls, the error is given compared to the results obtained with UWVF+MLFMM on 2S025. Indeed, in the case of scattering from two spheres, we consider our solution on $2 \mathrm{~S} 025$ as the reference solution. 
Table 5: Computational costs comparison for the basic UWVF (2S400; 2S200) and the UWVF+MLFMM (2S025; 4S025) for scattering from multiple objects.

\begin{tabular}{|c||c|c|c||c|c|c|c|c|c|}
\hline Case & T00 & T0c & T0f & TD & TC & TCc & TCf & Tcg & Nit \\
\hline \hline 2S400 & 250 & - & - & 1.12 & 7.4 & - & - & 1172 & 136 \\
\hline 2S200 & 93.5 & - & - & 0.5 & 2.68 & - & - & 424 & 130 \\
\hline 2S025 & 17.29 & 187 & 5.73 & 0.17 & 0.52 & 1.28 & 7.25 & 1087 & 118 \\
\hline \hline $4 \mathrm{~S} 025$ & 28 & 361 & 12.25 & 0.25 & 0.79 & 2.45 & 16.03 & 1675 & 86 \\
\hline
\end{tabular}

\begin{tabular}{|c||c|c||c|c|c|}
\hline Case & Ttot & mem & Rel Err 2 & Rel Err $\infty$ & RMS error \\
\hline \hline 2S400 & 1422 & 6.12 & $4.3 \quad 10^{-2}$ & $5.710^{-2}$ & $3.6 \quad 10^{-1}$ \\
\hline 2S200 & 518 & 2.28 & $7.810^{-2}$ & $9.910^{-2}$ & $6.4 \quad 10^{-1}$ \\
\hline 2S025 & 1297 & 3.34 & - & - & - \\
\hline \hline $4 \mathrm{~S} 025$ & 2077 & 6.73 & - & - & - \\
\hline
\end{tabular}
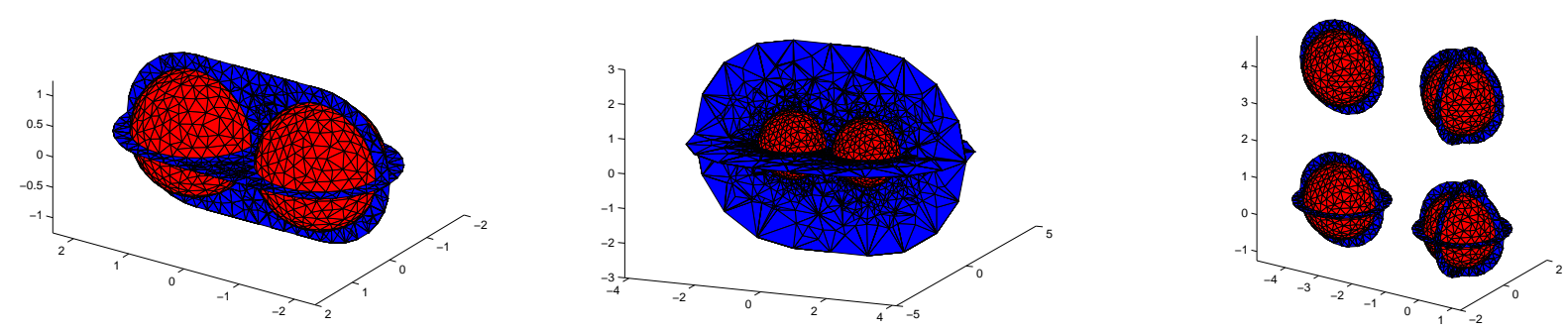

Figure 9: Meshes 2S025, 2S200 and 4S025.

The second example (results in Figure 11) is a set of four unit spheres centered at $( \pm 1.8,0, \pm 1.8)$, and the mesh is called $4 \mathrm{~S} 025$. In this case, the spheres are well separated and so we can take the artificial boundary to consist of four spheres of radius $1.5 \mathrm{~m}$ and centered at the same points as the spheres which contribute to the scatterer. In this situation, the domain is a four-components domain, and multiple scattering is taken into account by the integral equation. Figure 9 shows the domain. No error estimate is available for the case of scattering by four balls (mesh 4S025).

\section{Conclusion}

We have described how to couple the UWVF and multi-level fast multipole scheme via an overlapping strategy. This allows us to use non-convex artificial boundaries close to the scatterer, and hence decrease the memory and CPU requirements for solving an exterior scattering problem. The main content of the paper is a series of numerical tests that 1) demonstrate that the new method improves on the original UWVF when applied to simple scatterers, 2) supports the predicted wave number dependence of the work estimates and 3) shows how the method can handle multiple scatterers.

Clearly much more work is needed to make this method generally applicable. Error estimates should be proved and a better preconditioner for the UWVF derived. In addition a subgrid boundary model needs to be implemented to allow for a coarser volume grid near the boundary, and to allow a better approximation of curved boundaries. 

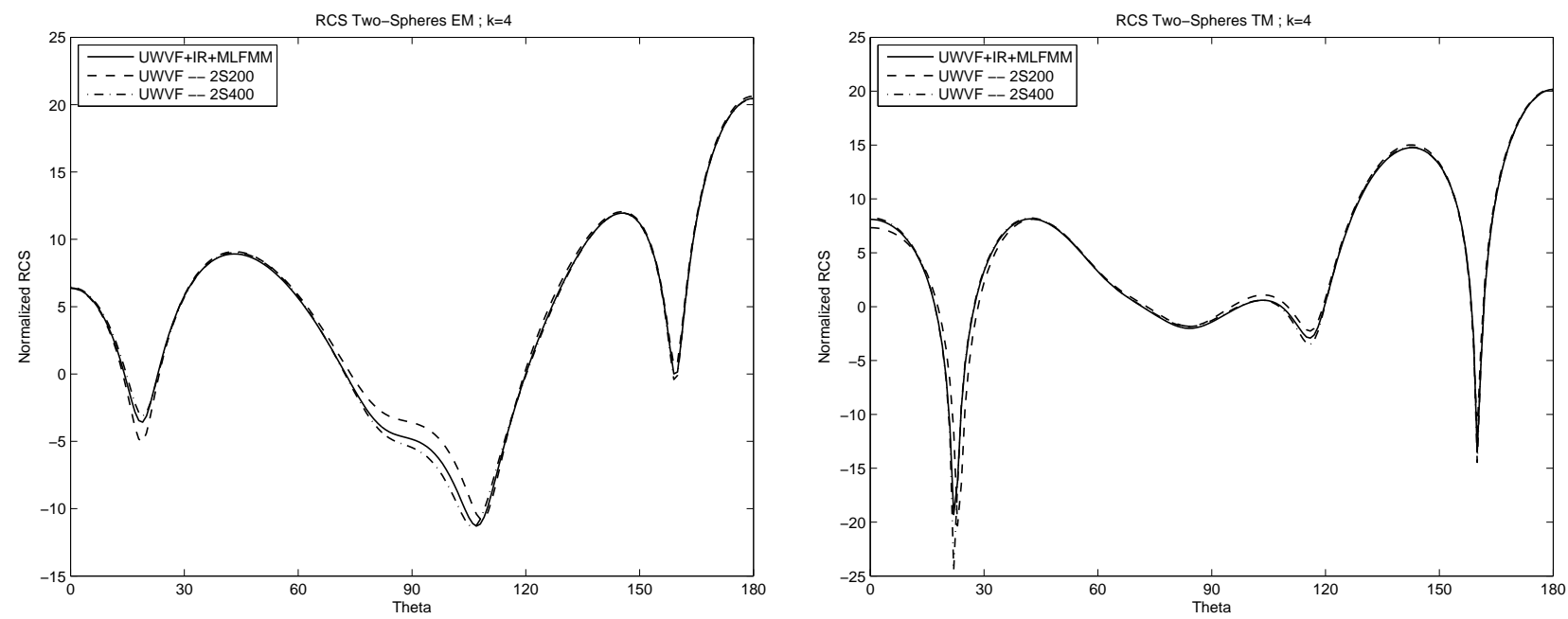

Figure 10: The RCS computed using UWVF+MLFMM with the mesh $2 \mathrm{~S} 025$ and using UWVF with the meshes 2S200 and 2S400, with $\kappa=4$. Left: TE-polarized RCS. Right: TM-polarized RCS.
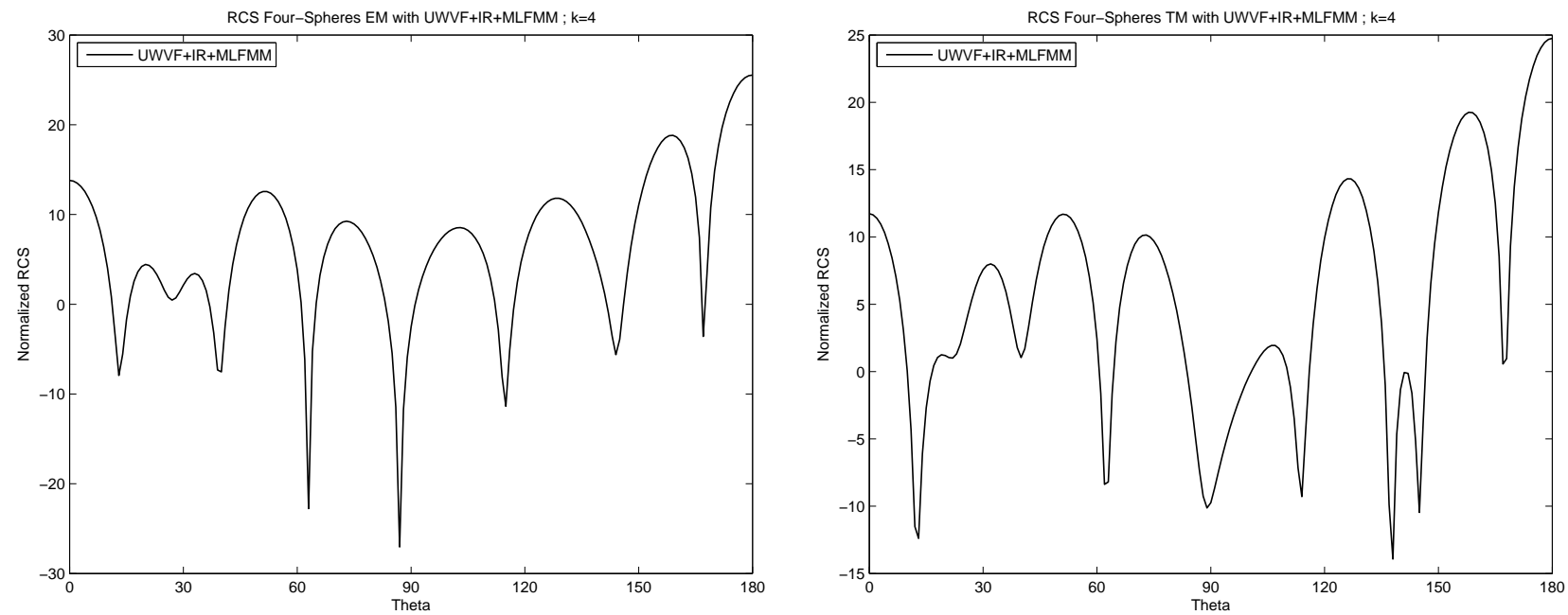

Figure 11: The RCS computed using UWVF+MLFMM with the mesh 4S025, with $\kappa=4$. Left: TE-polarized RCS. Right: TM-polarized RCS. 


\section{References}

[1] T. Abboud, J.-C. Nédélec and B. Zhou, "Improvement of the Integral Equation Method for High Frequency Problems", Third international conference on mathematical aspects of wave propagation phenomena, SIAM, pp. 178-187, 1995.

[2] O. Cessenat, Application d'une nouvelle formulation variationnelle aux équations d'ondes harmoniques problèmes de Helmholtz 2D et de Maxwell 3D, PhD-thesis, Université Paris IX, December 1996.

[3] O. Cessenat and B. Després, "Using Plane Waves as Base Functions for Solving Time Harmonic Equations with the Ultra Weak Variational Formulation", J. Comput. Acoust., vol. 11, pp. 227-238, 2003.

[4] R. Coifman, V. Rokhlin and S. Wandzura, "The Fast Multipole Method for the Wave Equation: A Pedestrian Prescription", IEEE Antennas and Propagation Magazine, vol. 35 (3), pp. 7-12, June 1993.

[5] D. Colton and R. Kress, Inverse Acoustic and Electromagnetic Scattering Theory, 2nd ed., Springer Verlag, New York, 1998.

[6] E. Darrigrand, "Coupling of Fast Multipole Method and Microlocal Discretization for the 3-D Helmholtz Equation", J. Comput. Phys., vol. 181 (1), pp. 126-154, September 2002.

[7] E. Darrigrand, "Ultra-Weak Variational Formulation and Efficient Integral Representation in Electromagnetism: a Thorough Study of the Algorithm Complexity", ESAIM: Proceedings, vol. 22, pp 190-197, 2007.

[8] E. Darrigrand and P. Monk, "Coupling of the Ultra-Weak Variational Formulation and an Integral Representation using a Fast Multipole Method in Electromagnetism", J. Comput. and Applied Math., vol. 204(2), pp 400-407, 2007.

[9] E. Darve, "The Fast Multipole Method: Numerical Implementation", J. Comput. Phys., vol. 160 (1), pp. 195-240, 2000.

[10] C. Hazard and M. Lenoir, "On the Solution of Time-Harmonic Scattering Problems for Maxwell's Equations", SIAM J. Math. Anal., vol. 6, pp. 1597-1630, November 1996.

[11] G. Hsiao, P. Monk and N. Nigam, "Error analysis of a finite element-integral equation scheme for approximating the time-harmonic Maxwell system", SIAM J. Numer. Anal., vol 40, pp. 198-219, 2002.

[12] T. Huttunen, M. Malinen and P.B. Monk, Solving Maxwell's Equations Using the Ultra Weak Variational Formulation, J. Comput. Phys., vol. 223, pp. 731-758, 2007

[13] J. Liu and J.M. Jin, "A Novel Hybridization of Higher Order Finite Element and Boundary Integral Methods for Electromagnetic Scattering and Radiation Problems", IEEE Trans. Ant. Prop., vol. 49, pp. 1794-1806, December 2001.

[14] J.M. Song and W.C. Chew, "Multilevel Fast Multipole Algorithm for Solving Combined Field Integral Equations of Electromagnetic Scattering", Microwave Opt. Tech. Letter, vol. 10, pp. 14-19, September 1995.

[15] T.J. Cui, W.C. Chew, G. Chen, and J. Song. Efficient MLFMA, RPFMA, and FAFFA algorithms for EM scattering by very large structures. IEEE Trans. on Antennas and Propag., vol. 52 (3), pp. 759-770, 2004. 